\title{
Neuroleptische Dickmacher
}

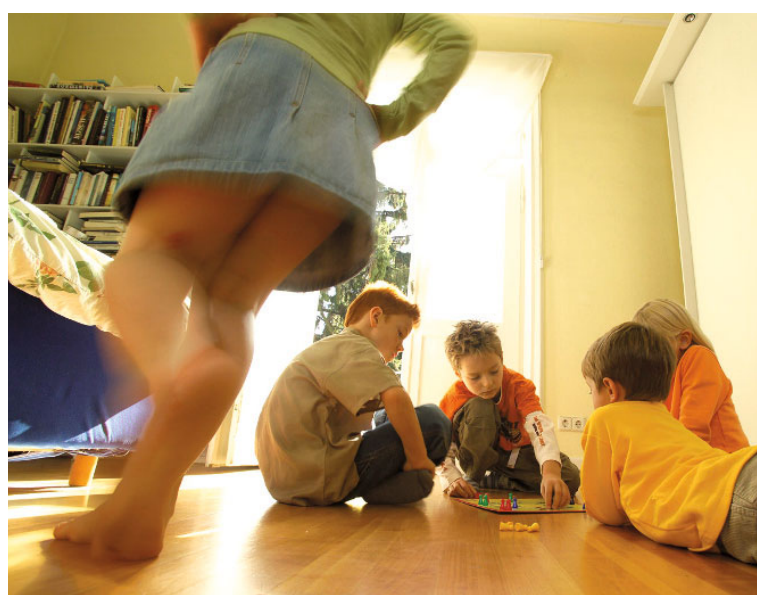

$\triangle$ Brauchen Kinder gleich Neuroleptika, wenn sie ein bisschen wild sind?

ridon um 5,3 $\mathrm{kg}$ und 41 unter Aripiprazol um $4,4 \mathrm{~kg}$. In der Vergleichs-

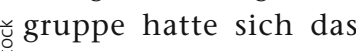
Ư̆ Körpergewicht nur um 은 einen Bereich zwischen 约 $-1,0$ und $+1,4 \mathrm{~kg}$ verän$\sum_{\text {un }}$ dert.

Unter Olanzapin und Quetiapin nahmen die mittleren Gesamtcholesterin-Konzentratio-

Die meisten der ZweitgenerationsNeuroleptika führen bei Kindern und Jugendlichen bereits in kurzer Zeit zu einer massiven Gewichtszunahme und einer ungünstigen Entwicklung metabolischer Parameter.

- Vor allem in den USA werden Neuroleptika mittlerweile im großen Umfang auch bei Kindern und Jugendlichen eingesetzt, darunter nicht nur für die Indikation Schizophrenie, sondern auch bei Autismus, bipolaren Störungen, erhöhter Aggressionsbereitschaft und Tics.

Zwischen 2003 und 2004 endete $1 \%$ der Konsultationen in pädiatrischen Praxen mit der Verordnung eines atypischen Neuroleptikums. In einer prospektiven Studie wurden das Körpergewicht und die metabolischen Parameter bei 505 Jugendlichen zwischen 4 und 19 Jahren untersucht, die erstmals mit Aripiprazol, Olanzapin, Quetiapin oder Risperidon behandelt wurden. 338 Jugendliche $(66,9 \%)$ konnten in die Studie einbezogen werden, 205 beendeten sie wie vorgesehen. 15 Patienten, welche die Therapie verweigerten, dienten als Vergleichsgruppe.

Nach einer medianen Behandlungsdauer von 10,8 Wochen hatten $45 \mathrm{Ju}$ gendliche unter Olanzapin im Durchschnitt 8,5 kg zugenommen, 36 unter Quetiapin um 6,1 kg, 135 unter Rispe- nen signifikant um 15,1 bzw. 9,1 mg/ dl zu, die Triglyzeride stiegen um 24,3 bzw. 37,0 mg/dl an. Risperidon hatte lediglich einen Einfluss auf die Triglyzeride, Aripiprazol war bezüglich der Stoffwechselparameter weitgehend neutral. Zwischen 10\% und 36\% aller Patienten entwickelten in einer Behandlungszeit von nur elf Wochen ein manifestes Übergewicht bzw. eine Adipositas.

KOMMENTAR: Man kann der Untersuchung vorwerfen, dass es sich um einen nicht randomisierten Ansatz mit bloßer Beobachtung handelt, die Dosis und eine evtl. Komedikation nicht berücksichtigt wurde, die Behandlungszeit sehr kurz und die Vergleichsgruppe sehr klein sei. Dennoch beeindruckt die Studie allein durch die große Anzahl behandelter Jugendlicher und einen Ansatz, wie er den realen Praxisbedingungen entspricht. Noch wissen wir nicht, welche Auswirkungen die Induktion von Übergewicht bei Kindern und Jugendlichen durch Neuroleptika auf das spätere Leben haben wird. Die Ergebnisse lassen es aber als angezeigt erscheinen, die Indikation für die Therapie von Kindern und Jugendlichen mit Zweitgenerations-Neuroleptika sehr hoch zu hängen.

H. S. FüEßL =

- C. U. Correll et al.

Cardiometabolic risk of second-generation antipsychotic medications during first-time use in children and adolescents. JAMA 302 (2009) 16, 1765-1773 